\title{
$p$-Adic Heisenberg Group and Maslov Index
}

\author{
E. I. Zelenov \\ Steklov Mathematical Institute, Vavilov str. 42, GSP-1, 117966 Moscow, Russia
}

Received October 29, 1991; in revised form October 22, 1992

\begin{abstract}
A "system of coordinates" on a set $\Lambda$ of selfdual lattices in a twodimensional $p$-adic symplectic space $(\mathscr{V}, \mathscr{B})$ is suggested. A unitary irreducible representation of the Heisenberg group of the space $(\mathscr{V}, \mathscr{B})$ depending on a lattice $\mathscr{L} \in \Lambda$ (an analogue of the Cartier representation) is constructed and its properties are investigated. By the use of such representations for three different lattices $\mathscr{L}_{1}, \mathscr{L}_{2}, \mathscr{L}_{3} \in \Lambda$ one defines the Maslov index $\mu=\mu\left(\mathscr{L}_{1}, \mathscr{L}_{2}, \mathscr{B}_{3}\right)$ of a triple of lattices. Properties of the index $\mu$ are investigated and values of $\mu$ in coordinates for different triples of lattices are calculated.
\end{abstract}

\section{Introduction}

As it is known one of the profitable methods to study a quantization procedure is to construct and to investigate topological characteristics associated with this procedure. An example of such a characteristic is the Maslov index [Ma]. Let us discuss generally one way to obtain such characteristics. Let $G$ be a group and $\left(H_{i}, U_{\imath}\right), i=1,2,3$ be its unitary irreducible representations in the Hilbert spaces $H_{i}, i=1,2,3$ respectively. Let us assume that these representations are unitary equivalent and $F_{21}, F_{32}$ and $F_{13}$ be unitary intertwining operators. That is, say for $F_{21}, F_{21}: H_{1} \rightarrow H_{2}$ and for all $g \in G$ the relation

$$
F_{21}^{-1} U_{2}(g) F_{21}=U_{1}(g)
$$

holds (and similarly for operators $F_{32}$ and $F_{13}$ ). By the last formula the operator $F=F_{13} F_{32} F_{21}: H_{1} \rightarrow H_{1}$ commutes with all operators $U_{1}(g), g \in G$. In view of irreducibility of $\left(H_{1}, U_{1}\right)$ the operator $F$ is proportional to the identity operator, that is $F=\mu$ Id for some $\mu \in \mathbb{T}$ ( $\mathbb{T}$ denotes a unit circle in the field $\mathbb{C}$ of complex numbers). Hence we obtain a numerical characteristic $\mu$ of a group $G$ and a triple of its unitary irreducible representations. 
Let us take an example, see [LV]. Let $(\mathscr{V}, \mathscr{B})$ be a two-dimensional symplectic vector space over the field $\mathbb{R}$ of real numbers and $\tilde{\mathscr{V}}$ be the Heisenberg group of the space $(\mathscr{V}, \mathscr{B})$ (that is $\tilde{\mathscr{V}}$ is the three-dimensional Heisenberg group). Let also $L$ be a lagrangian (that is one-dimensional for $\operatorname{dim} \mathscr{V}=2$ ) subspace of $\mathscr{V}$ provided with the natural Haar measure $d m(L)$. As it is known there is a unitary irreducible representation $\left(H(L), U_{L}\right)$ of the group $\tilde{\mathscr{V}}$ in the Hilbert space $H(L)=L^{2}(L, d m(L))$. For two different lagrangian subspaces $L_{1}$ and $L_{2}$ these representations are unitary equivalent. Let now $L_{1}, L_{2}$ and $L_{3}$ be different lagrangian subspaces in $\mathscr{\mathscr { V }}$. By applying the procedure discussed above for the group $\tilde{\mathscr{V}}$ and for the representations $U_{L_{1}}, U_{L_{2}}$ and $U_{L_{3}}$ we obtain a numerical characteristic $\mu\left(L_{1}, L_{2}, L_{3}\right)$ of these representations. It turns out that in this case $\mu=\exp (i \pi \tau / 4)$, where $\tau=\tau\left(L_{1}, L_{2}, L_{3}\right) \in \mathbb{Z}$ is the Maslov index of lagrangian subspaces $L_{1}, L_{2}$ and $L_{3}$, see [LV].

As a different example we consider the Cartier representation [C] of the Heisenberg group $\tilde{\mathscr{V}}$. This representation is unitary, irreducible and depends on a selfdual $\mathbb{Z}$-lattice $\mathscr{L}$ in the space $\mathscr{V}$. By using the procedure discussed above for the Cartier representations associated with lattices $\mathscr{L}_{1}, \mathscr{L}_{2}$ and $\mathscr{L}_{3}$ we obtain an index of a triple $\left(\mathscr{L}_{1}, \mathscr{L}_{2}, \mathscr{L}_{3}\right)$ of selfdual $\mathbb{Z}$-lattices, see [LV].

As $p$-adic numbers find expanding applications in mathematical physics (the active advancement began from the paper [V]) it is interesting to extend the construction discussed above for the field $\mathbb{Q}_{p}$ of $p$-adic numbers. Let now $(\mathscr{V}, \mathscr{B})$ be a twodimensional symplectic vector space over $\mathbb{Q}_{p}$ and $\tilde{\mathscr{V}}$ be the Heisenberg group of this space (for the definition of the group $\tilde{\mathscr{V}}$ see Sect. 3 of this paper). As for the field $\mathbb{R}$ there is a unitary irreducible representation of $\tilde{\mathscr{V}}$ in the space $L^{2}(L, d m(L))$, where $L$ is a lagrangian subspace of the space $\mathscr{V}$ and $d m(L)$ is the Haar measure on $L$, as to the corresponding index see $[\mathrm{LV}]$ and bibliography there.

There exist also a unitary irreducible representation of the $p$-adic Heisenberg group depending on a selfdual $\mathbb{Z}_{p}$-lattice in the space $\mathscr{V} .\left(\mathbb{Z}_{p}\right.$ denotes a ring of $p$-adic integers.) This representation is an analogue of the Cartier representation mentioned above. By applying the procedure discussed above for the $p$-adic Heisenberg group and a triple of its representations associated with lattices $\mathscr{L}_{1}, \mathscr{L}_{2}$ and $\mathscr{L}_{3}$ we obtain a complex number $\mu=\mu\left(\mathscr{L}_{1}, \mathscr{L}_{2}, \mathscr{L}_{3}\right) \in \mathbb{T}$. This number $\mu$ we call the Maslov index of a triple $\left(\mathscr{L}_{1}, \mathscr{L}_{2}, \mathscr{B}_{3}\right)$ of selfdual $\mathbb{Z}_{p}$-lattices. This index is the subject of our investigation. It is not improbable that this index will be useful for $p$-adic quantum mechanics constructed in [VV] (see also [Me, R]).

The structure of this paper is the following. In Sect. 2 one considers $\mathbb{Z}_{p}$-lattices and their properties. In particular one constructs a "system of coordinates" on a set $\Lambda$ of selfdual $\mathbb{Z}_{p}$-lattices in a two-dimensional symplectic space $(\mathscr{V}, \mathscr{B})$ over $\mathbb{Q}_{p}$ (Proposition 1). In Sect. 3 we define the Heisenberg group $\tilde{\mathscr{V}}$ of the space $(\mathscr{V}, \mathscr{B})$ and construct a unitary irreducible representation $\left(H(\mathscr{L}), W_{\mathscr{L}}\right)$ of this group depending on a lattice $\mathscr{L} \in \Lambda$. We prove also some properties of this representation (Proposition 2). In Sect. 4 an intertwining operator of two such representation is constructed and its properties are investigated (Proposition 3). In Sect. 5 we construct the Maslov index of a triple of selfdual $\mathbb{Z}_{p}$-lattices. We also obtain an explicit formula for this index (Proposition 4) and prove some natural properties of the index (Proposition 5). Section 6 is devoted to calculations of the Maslov index in coordinates defined in Sect. 2. 


\section{Lattices}

Let $(\mathscr{T}, \mathscr{S})$ be a two dimensional symplectic space over $\mathbb{Q}_{p}$ and $\mathscr{L}$ be a lattice in $(\mathscr{T}, \mathscr{B})$ (that is $\mathscr{S}$ is a finitely generated $\mathbb{Z}_{p}$-submodule of the space $\mathscr{V}$ containing a basis of $\mathscr{T}$ ). A dual lattice $\mathscr{L}^{*}$ is defined as follows:

$$
\mathscr{E}^{*}=\left\{x \in \mathscr{T}: \mathscr{B}(x, y) \in \mathbb{Z}_{p} \forall y \in \mathscr{L}\right\} \text {. }
$$

If $\mathscr{L}=\mathscr{S}^{*}$, then $\mathscr{B}$ is a selfdual lattice. Let $\Lambda=\Lambda(\mathscr{T}, \mathscr{B})$ denote the set of all selfdual lattices in $(\mathscr{T}, \mathscr{B})$. Note that if $\mathscr{S} \in \Lambda(\mathscr{W}, \mathscr{B})$, then $(\mathscr{L}, \mathscr{B})$ is a space with symplectic inner product.

As $\mathbb{Z}_{p}$ is a local ring, then there exists a symplectic basis $\{e, f\}$ of the space $(\mathscr{T}, \mathscr{乃})$ (symplectic means that $\mathscr{B}(e, f)=1$ ) wherein (see $[\mathrm{MH}]$ )

$$
\mathscr{L}=\mathbb{Z}_{p} e \oplus \mathbb{Z}_{p} f
$$

Moreover for any $\mathscr{L}_{1}, \mathscr{S}_{2} \in \Lambda$ there is a symplectic basis $\{e, f\}$ wherein these lattices have the form

$$
\mathscr{L}_{1}=\mathbb{Z}_{p} e \oplus \mathbb{Z}_{p} f, \quad \mathscr{L}_{2}=p^{m} \mathbb{Z}_{p} e \oplus p^{-m} \mathbb{Z}_{p} f
$$

for some nonnegative integer $m$. For the proof of existence of such basis (but is not of necessity symplectic) see for example [W1], reduction to symplectic case is rather obvious.

Now we define a "system of coordinates" on the set $\Lambda$. Let $\operatorname{Sp}(\mathscr{V})$ denote the group of all linear automorphisms of $\mathscr{V}$ preserving the form $\mathscr{B}$ (symplectic group) and $S p(\mathscr{S})$ be a stabilizer of a selfdual lattice $\mathscr{S}$ in $S p(\mathscr{T}) . S p(\mathscr{T})$ acts on $\Lambda$ in a standard manner, this action is transitive. Thus $\Lambda$ can be identified with the homogeneous space $S p(\mathscr{T}) / S p(\mathscr{L})$.

Proposition 1. Let $\{e, f\}$ be a symplectic basis in $(\mathscr{T}, \mathscr{B})$. Then the map $\varphi: \mathbb{Z} \times$ $\mathbb{Q}_{p} / \mathbb{Z}_{p} \rightarrow \Lambda$

$$
\mathbb{Z} \times \mathbb{Q}_{p} / \mathbb{Z}_{p} \ni(m, \bar{\mu}) \stackrel{\varphi}{\mapsto} \mathbb{Z}_{p} p^{m} e \oplus \mathbb{Z}_{p}\left(\mu p^{m} e+p^{-m} f\right) \in \Lambda
$$

defines a one-to-one correspondence between $\mathbb{Z} \times \mathbb{Q}_{p} / \mathbb{Z}_{p}$ and $\Lambda$. (In the right-hand part of the last formula $\mu$ denotes an arbitrary element of a coset $\bar{\mu}$.)

Proof. Let $\mathscr{L}_{0}$ denote the following lattice:

$$
\mathscr{S}_{0}=\mathbb{Z}_{p} e+\mathbb{Z}_{p} f
$$

In the basis $\{e, f\} S p(\mathscr{T})$ and $S p\left(\mathscr{C}_{0}\right)$ have the matrix realizations: $S p(\mathscr{T}) \cong$ $S L\left(2, \mathbb{Q}_{p}\right), S p\left(\mathscr{S}_{0}\right) \cong S L\left(2, \mathbb{Z}_{p}\right)$. Let $\mathscr{L}$ be an arbitrary lattice from $\Lambda$. Then there is an element $g \in S L\left(2, \mathbb{Q}_{p}\right)$ such that $\mathscr{L}=g \mathscr{L}_{0}$. By the Iwasawa decomposition (see $[\mathrm{PR}]) g$ can be represented in the form:

$$
g=\left(\begin{array}{cc}
p^{m} & 0 \\
0 & p^{-m}
\end{array}\right)\left(\begin{array}{cc}
1 & \mu \\
0 & 1
\end{array}\right) g_{0}
$$

for some $m \in \mathbb{Z}, \mu \in \mathbb{Q}_{p}$ and $g_{0} \in S L\left(2, \mathbb{Z}_{p}\right)$. Thus $\mathscr{L}$ has the form

$$
\mathscr{L}=\mathbb{Z}_{p} p^{m} e \oplus \mathbb{Z}_{p}\left(\mu p^{m} e+p^{-m} f\right)
$$


and the map $\varphi$ is surjective. As for $m, m^{\prime} \in \mathbb{Z}$ and $\mu, \mu^{\prime} \in \mathbb{Q}_{p}$ we have

$$
\begin{gathered}
{\left[\left(\begin{array}{cc}
p^{m^{\prime}} & 0 \\
0 & p^{-m^{\prime}}
\end{array}\right)\left(\begin{array}{cc}
1 & \mu^{\prime} \\
0 & 1
\end{array}\right)\right]^{-1}\left(\begin{array}{cc}
p^{m} & 0 \\
0 & p^{-m}
\end{array}\right)\left(\begin{array}{ll}
1 & \mu \\
0 & 1
\end{array}\right)} \\
=\left(\begin{array}{cc}
p^{m-m^{\prime}} & p^{m-m^{\prime}} \mu-p^{m^{\prime}-m} \mu^{\prime} \\
0 & p^{m^{\prime}-m}
\end{array}\right) \in S L\left(2, \mathbb{Z}_{p}\right)
\end{gathered}
$$

if and only if $m=m^{\prime}$ and $\mu-\mu^{\prime} \in \mathbb{Z}_{p}$, then the definition of the map $\varphi$ is correct (that is it doesn't depend on a choice of $\mu$ in a coset $\bar{\mu}$ ). This finishes the proof.

Corollary. For any $\mathscr{L}_{1}, \mathscr{L}_{2}, \mathscr{L}_{3} \in \Lambda$ there is a symplectic basis $\{e, f\}$ wherein

$$
\begin{aligned}
& \mathscr{L}_{1}=\mathbb{Z}_{p} e \oplus \mathbb{Z}_{p} f, \\
& \mathscr{L}_{2}=p^{m} \mathbb{Z}_{p} e \oplus p^{-m} \mathbb{Z}_{p} f \\
& \mathscr{L}_{3}=\mathbb{Z}_{p} p^{n} e \oplus \mathbb{Z}_{p}\left(\nu p^{n} e+p^{-n} f\right)
\end{aligned}
$$

for some $m \in \mathbb{Z}_{\geq 0}, n \in \mathbb{Z}, \nu \in \mathbb{Q}_{p}$.

\section{3. -Adic Heisenberg Group}

Let $\chi_{p}$ be an additive character of $\mathbb{Q}_{p}$ of rank 0 (that is $\chi_{p}(x)=1$ if and only if $\left.x \in \mathbb{Z}_{p}\right), \mathbb{T}$ be a unit circle in the field $\mathbb{C}$ of complex numbers. Heisenberg group $\tilde{\mathscr{V}}$ of a space $(\mathscr{V}, \mathscr{B})$ is the set of pairs

$$
\tilde{\mathscr{V}}=\{(\alpha, x), \alpha \in \mathbb{T}, x \in \mathscr{V}\}
$$

with the composition law

$$
(\alpha, x)(\beta, y)=\left(\alpha \beta \chi_{p}(1 / 2 \mathscr{\beta}(x, y)), x+y\right) .
$$

We assume that $p \neq 2$ below. Now we construct some representation of $\tilde{\mathscr{V}}$. This representation depends on a lattice $\mathscr{L} \in \Lambda$ and therefore we call it $\mathscr{L}$-representation. Let $\tilde{H}(\mathscr{L})$ denote the space of finite complex valued functions on $\mathscr{V}$ satisfying the relation

$$
f(x+u)=\chi_{p}(1 / 2 \mathscr{B}(x, u)) f(x)
$$

for all $x \in \mathscr{V}$ and $u \in \mathscr{L}$. Note that if $f, g \in \tilde{H}(\mathscr{B})$ then $|f|$ and $f \bar{g}$ are constant on every coset in $\mathscr{T} / \mathscr{L}$ and nonzero only on a finite number of such cosets. For $f, g \in \tilde{H}(\mathscr{L})$ the formula

$$
(f, g)=\sum_{\alpha \in \mathscr{T} / \mathscr{G}} f(\alpha) \bar{g}(\alpha)
$$

defines a nonnegative hermitian form on $\tilde{H}(\mathscr{L})$ and thus $\tilde{H}(\mathscr{C})$ is provided by a prehilbertian structure. The space $H(\mathscr{B})$ of $\mathscr{B}$-representation is defined as the completion of $\tilde{H}(\mathscr{L})$ with respect to the norm $\|\cdot\|^{2}=(\cdot, \cdot)$. As $\mathscr{T} / \mathscr{L}$ is a countable set, then $H(\mathscr{L})$ is a separable Hilbert space.

On the space $\tilde{H}(\mathscr{L})$ we define the following set of operators, $x, y \in \mathscr{V}$ :

$$
\left(W_{\mathscr{S}}(x) f\right)(y)=\chi_{p}(1 / 2 \mathscr{B}(x, y)) f(y-x) .
$$


These operators satisfy the co-called Weyl relation

$$
W_{\mathscr{L}}(x) W_{\mathscr{C}}(y)=\chi_{p}(1 / 2 \mathscr{B}(x, y)) W_{\mathscr{C}}(x+y) .
$$

It is easy to see that $W_{\mathscr{C}}(x), x \in \mathscr{T}$ are isometric operators on $\tilde{H}(\mathscr{B})$ and therefore are uniquely extended to unitary operators on $H(\mathscr{L})$ (for these operators we retain the same notation $\left.W_{\mathscr{L}}(x)\right)$. $\mathscr{L}$-representation of $\tilde{\mathscr{V}}$ is defined as a pair $\left(H(\mathscr{L}), \tilde{W}_{\mathscr{L}}\right)$, where $\tilde{W}_{\mathscr{L}}(\alpha, x)=\alpha W_{\mathscr{L}}(x)$. From the Weyl relation we see that this pair is in fact a unitary representation of $\tilde{\mathscr{V}}$. For the sake of convenience we use the term " $\mathscr{L}$ representation" for a pair $\left(H(\mathscr{L}), W_{\mathscr{C}}(x)\right)$. A similar representation was considered in [W2]. Note that $\mathscr{L}$-representation is a $p$-adic analogue of the Cartier representation $[C]$ of the Heisenberg group over real numbers.

Let $\phi_{\mathscr{C}}$ denote the following element of $H(\mathscr{C})$ :

$$
\phi_{\mathscr{S}}(u)= \begin{cases}1, & u \in \mathscr{L}, \\ 0, & u \notin \mathscr{B} .\end{cases}
$$

We call it a vacuum vector of $\left(H(\mathscr{D}), W_{\mathscr{L}}(x)\right)$. It is easy to see that this vector satisfies the property

$$
W_{\mathscr{C}}(x) \phi_{\mathscr{L}}=\phi_{\mathscr{E}}
$$

for all $x \in \mathscr{B}$.

Let $\eta_{\mathscr{C}}: \mathscr{V} \rightarrow \mathbb{T}$ be a function satisfying the property

$$
\eta_{\mathscr{C}}(x+u)=\chi_{p}(1 / 2 \mathscr{B}(x, u)) \eta_{\mathscr{E}}(x)
$$

for all $x \in \mathscr{V}$ and $u \in \mathscr{L}$. It is quite easy to prove that the map $\mathscr{V} \rightarrow H(\mathscr{L})$ :

$$
\mathscr{V} \ni x \mapsto \eta_{\mathscr{E}}(x) W_{\mathscr{L}}(x) \phi_{\mathscr{L}}
$$

is constant on every coset in $\mathscr{V} / \mathscr{C}$ and thus one defines a map $\psi: \mathscr{V} / \mathscr{L} \rightarrow H(\mathscr{L})$ by the same formula. The range of values of the map $\psi$ we call a set of coherent states of $\mathscr{L}$-representation.

Proposition 2. The representation $\left(H(\mathscr{S}), W_{\mathscr{G}}(x)\right)$ has the properties:

(i) $\left(W_{\mathscr{L}}(x) \phi_{\mathscr{C}}, \phi_{\mathscr{C}}\right)=\phi_{\mathscr{C}}(x)$;

(ii) the set of coherent states forms an orthonormal basis in $H(\mathscr{L})$;

(iii) the representation $\left(H(\mathscr{L}), W_{\mathscr{L}}(x)\right)$ is irreducible.

\section{Intertwining Operator}

Let for $\mathscr{L}_{1}, \mathscr{L}_{2} \in \Lambda \varrho^{-2}\left(\mathscr{L}_{1}, \mathscr{L}_{2}\right)$ denotes the number of elements of the group $\mathscr{L}_{1} /\left(\mathscr{L}_{1} \cap \mathscr{L}_{2}\right)$.

Proposition 3. Let $\left(H\left(\mathscr{L}_{1}\right), W_{\mathscr{L}_{1}}\right)$ and $\left(H\left(\mathscr{C}_{2}\right), W_{\mathscr{L}_{2}}\right)$ be $\mathscr{L}_{1}$ - and $\mathscr{L}_{2}$-representations. Then the operator $F_{\mathscr{L}_{2}, \mathscr{L}_{1}}: H\left(\mathscr{S}_{1}\right) \rightarrow H\left(\mathscr{L}_{2}\right)$ defined by the formula

$$
F_{\mathscr{L}_{2}, \mathscr{K}_{1}} f(u)=\varrho\left(\mathscr{L}_{1}, \mathscr{L}_{2}\right) \sum_{\alpha \in \mathscr{L}_{2} /\left(\mathscr{L}_{1} \cap \mathscr{L}_{2}\right)} \chi_{p}(1 / 2 \mathscr{B}(\alpha, u)) f(u+\alpha)
$$

is a unitary operator. It satisfies the property

$$
F_{\mathscr{C}_{2}, \mathscr{L}_{1}}^{-1}=F_{\mathscr{L}_{1}, \mathscr{L}_{2}}
$$


and it is an intertwining operator for the $\mathscr{L}_{1}$ - and $\mathscr{L}_{2}$-representations, that is for all $x \in \mathscr{V}$ the following relation holds:

$$
F_{\mathscr{L}_{2}, \mathscr{L}_{1}}^{-1} W_{\mathscr{L}_{2}}(x) F_{\mathscr{L}_{2}, \mathscr{L}_{1}}=W_{\mathscr{L}_{1}}(x)
$$

Proof. At first we check that the definition (1) is correct, that is the right-hand part of the formula (2) doesn't depend on a choice of an element in coset $\alpha \in \mathscr{L}_{1} /\left(\mathscr{L}_{1} \cap \mathscr{L}_{2}\right)$. In fact taking into account that $f \in H\left(\mathscr{L}_{1}\right)$ for $\alpha^{\prime} \in \mathscr{D}_{1} \cap \mathscr{L}_{2}$ we have

$$
\begin{aligned}
& \sum_{\alpha \in \mathscr{L}_{2} /\left(\mathscr{L}_{1} \cap \mathscr{L}_{2}\right)} \chi_{p}\left(1 / 2 \mathscr{B}\left(\alpha+\alpha^{\prime}, u\right)\right) f\left(u+\alpha+\alpha^{\prime}\right) \\
= & \sum_{\alpha \in \mathscr{L}_{2} /\left(\mathscr{L}_{1} \cap \mathscr{L}_{2}\right)} \chi_{p}\left(1 / 2 \mathscr{B}\left(\alpha+\alpha^{\prime}, u\right)+1 / 2 \mathscr{B}\left(u+\alpha, \alpha^{\prime}\right)\right) f(u+\alpha) \\
= & \sum_{\alpha \in \mathscr{L}_{2} /\left(\mathscr{L}_{1} \cap \mathscr{L}_{2}\right)} \chi_{p}\left(1 / 2 \mathscr{B}\left(\alpha, \alpha^{\prime}\right)\right) \chi_{p}(1 / 2 \mathscr{B}(\alpha, u)) f(u+\alpha) \\
= & \sum_{\alpha \in \mathscr{L}_{2} /\left(\mathscr{L}_{1} \cap \mathscr{L}_{2}\right)} \chi_{p}(1 / 2 \mathscr{B}(\alpha, u)) f(u+\alpha) .
\end{aligned}
$$

It is easy to check that for $f \in H\left(\mathscr{L}_{1}\right)$ the condition $F_{\mathscr{L}_{2}, \mathscr{L}_{1}} f \in H\left(\mathscr{L}_{2}\right)$ holds. get

Let us prove unitarity of $F_{\mathscr{L}_{2}, \mathscr{L}_{1}}$. From the definition of the operator $F_{\mathscr{L}_{2}, \mathscr{L}_{1}}$ we

$$
F_{\mathscr{L}_{2}, \mathscr{L}_{1}} f(u)=\varrho\left(\mathscr{L}_{1}, \mathscr{L}_{2}\right) \sum_{\alpha \in \mathscr{L}_{2} /\left(\mathscr{L}_{1} \cap \mathscr{L}_{2}\right)} \chi_{p}(\mathscr{B}(\alpha, u)) W_{\mathscr{L}_{1}}(-\alpha) f(u) .
$$

From the definition of $\mathscr{L}$-representation, orthogonality of coherent states, ParsevalStokes relation and the last formula we have

$$
\left\|F_{\mathscr{L}_{2}, \mathscr{L}_{1}} f\right\|_{H\left(\mathscr{L}_{2}\right)}^{2}=\varrho^{2}\left(\mathscr{L}_{1}, \mathscr{L}_{2}\right) \sum_{\alpha \in \mathscr{L}_{2} /\left(\mathscr{L}_{1} \cap \mathscr{L}_{2}\right)}\left\|W_{\mathscr{L}_{1}}(-\alpha) f\right\|_{H\left(\mathscr{L}_{1}\right)}^{2}=\|f\|_{H\left(\mathscr{L}_{1}\right)}^{2} .
$$

Now we prove the formula (3). Taking into account the condition $f \in H\left(\mathscr{L}_{1}\right)$ we get

$$
\begin{aligned}
F_{\mathscr{L}_{1}, \mathscr{L}_{2}} F_{\mathscr{L}_{2}, \mathscr{L}_{1}} f(u) & \varrho^{2}\left(\mathscr{L}_{1}, \mathscr{L}_{2}\right) \sum_{\beta \in \mathscr{L}_{1} /\left(\mathscr{L}_{1} \cap \mathscr{L}_{2}\right)} \chi_{p}(1 / 2 \mathscr{B}(\beta, u)) \\
& \times \sum_{\alpha \in \mathscr{L}_{2} /\left(\mathscr{L}_{1} \cap \mathscr{L}_{2}\right)} \chi_{p}(1 / 2 \mathscr{B}(\alpha, u+\beta)) f(u+\alpha+\beta) \\
= & \varrho^{2}\left(\mathscr{L}_{1}, \mathscr{L}_{2}\right) \sum_{\alpha \in \mathscr{L}_{2} /\left(\mathscr{L}_{1} \cap \mathscr{L}_{2}\right)} \\
& \times \chi_{p \in \mathscr{L}_{1} /\left(\mathscr{L}_{1} \cap \mathscr{L}_{2}\right)}(1 / 2 \mathscr{B}(\beta, u)+1 / 2 \mathscr{B}(\alpha, u+\beta)+1 / 2 \mathscr{B}(u+\alpha, \beta)) f(u+\alpha) \\
= & \varrho^{2}\left(\mathscr{L}_{1}, \mathscr{L}_{2}\right) \sum_{\alpha \in \mathscr{L}_{2} /\left(\mathscr{L}_{1} \cap \mathscr{L}_{2}\right)} \chi_{p}(1 / 2 \mathscr{B}(\alpha, u)) f(u+\alpha) \sum_{\beta \in \mathscr{L}_{1} /\left(\mathscr{L}_{1} \cap \mathscr{L}_{2}\right)} \chi_{p}(\mathscr{B}(\alpha, \beta))
\end{aligned}
$$


and (3) follows from the formula

$$
\varrho^{2}\left(\mathscr{L}_{1}, \mathscr{L}_{2}\right) \sum_{\beta \in \mathscr{L}_{1} /\left(\mathscr{C}_{1} \cap \mathscr{L}_{2}\right)} \chi_{p}(\mathscr{S}(\alpha, \beta))= \begin{cases}1, & \alpha \in \mathscr{L}_{1} \cap \mathscr{L}_{2}, \\ 0, & \alpha \notin \mathscr{L}_{2} \cap \mathscr{L}_{1} .\end{cases}
$$

For $\alpha \in \mathscr{L}_{1} \cap \mathscr{L}_{2}(6)$ obviously follows from the definition of $\varrho\left(\mathscr{L}_{1}, \mathscr{L}_{2}\right)$. For $\alpha \notin \mathscr{L}_{2} \cap \mathscr{L}_{1}$ let us choose $\beta^{\prime} \in \mathscr{L}_{1}$ satisfying the condition $\chi_{p}\left(\mathscr{\mathscr { S }}\left(\alpha, \beta^{\prime}\right)\right) \neq 1$ (by virtue of selfduality of $\mathscr{L}_{1}$ such $\beta^{\prime}$ always exists). Then

$$
\begin{aligned}
& \varrho^{2}\left(\mathscr{S}_{1}, \mathscr{L}_{2}\right) \sum_{\beta \in \mathscr{L}_{1} /\left(\mathscr{L}_{1} \cap \mathscr{L}_{2}\right)} \chi_{p}(\mathscr{B}(\alpha, \beta)) \\
& =\varrho^{2}\left(\mathscr{L}_{1}, \mathscr{L}_{2}\right) \sum_{\beta \in \mathscr{L}_{1} /\left(\mathscr{L}_{1} \cap \mathscr{L}_{2}\right)} \chi_{p}\left(\mathscr{B}\left(\alpha, \beta+\beta^{\prime}\right)\right) \\
& =\chi_{p}\left(\mathscr{B}\left(\alpha, \beta^{\prime}\right)\right) \varrho^{2}\left(\mathscr{L}_{1}, \mathscr{L}_{2}\right) \sum_{\beta \in \mathscr{L}_{1} /\left(\mathscr{L}_{1} \cap \mathscr{L}_{2}\right)} \chi_{p}(\mathscr{R}(\alpha, \beta))
\end{aligned}
$$

and therefore (6) is valid. The property (4) of the operator $F_{\mathscr{L}_{2}, \mathscr{L}_{1}}$ can be proved by analogy to that of (3).

The operator $F_{\mathscr{L}_{2}, \mathscr{L}_{1}}$ we call a canonical intertwining operator.

In particular from the last proposition it follows that $\mathscr{L}_{1}$ - and $\mathscr{L}_{2}$-representations are unitary equivalent.

\section{Maslov Index}

Let $\mathscr{L}_{1}, \mathscr{L}_{2}, \mathscr{L}_{3} \in \Lambda$. Then the corresponding representations $\left(H\left(\mathscr{L}_{1}\right), W_{\mathscr{L}_{1}}\right),\left(H\left(\mathscr{L}_{2}\right)\right.$, $\left.W_{\mathscr{L}_{2}}\right)$ and $\left(H\left(\mathscr{L}_{3}\right), W_{\mathscr{L}_{3}}\right)$ are unitary equivalent. Let us consider the unitary operator $\mathscr{F}=F_{\mathscr{L}_{1}, \mathscr{H}_{3}} F_{\mathscr{L}_{3}, \mathscr{L}_{2}} F_{\mathscr{L}_{2}, \mathscr{L}_{1}}$ on the space $H\left(\mathscr{L}_{1}\right)$. By using the formula (4) for intertwining operators $F_{\mathscr{L}_{1}, \mathscr{L}_{3}}, F_{\mathscr{L}_{3}, \mathscr{K}_{2}}$ and $F_{\mathscr{L}_{2}, \mathscr{K}_{1}}$ it is easy to see that the operator $\mathscr{F}$ commutes with all operators $W_{\mathscr{L} 1}(x), x \in \mathscr{V}$ and by virtue of irreducibility of the $\mathscr{S}_{1}$-representation $\left(H\left(\mathscr{S}_{1}\right), W_{\mathscr{L}_{1}}\right)$ it is proportional to the identity operator on $H\left(\mathscr{D}_{1}\right)$. Thus we have

$$
\mathscr{F}=\mu\left(\mathscr{S}_{1}, \mathscr{L}_{2}, \mathscr{L}_{3}\right) \mathrm{Id}
$$

The number $\mu\left(\mathscr{L}_{1}, \mathscr{L}_{2}, \mathscr{L}_{3}\right) \in \mathbb{T}$ we call the Maslov index of a triple of selfdual lattices.

Let us take an explicit formula for the Maslov index.

Proposition 4. Let $\mathscr{L}_{1}, \mathscr{L}_{2}, \mathscr{L}_{3} \in \Lambda$. Then the following formula holds:

$$
\mu\left(\mathscr{L}_{1}, \mathscr{L}_{2}, \mathscr{L}_{3}\right)=\frac{\varrho\left(\mathscr{L}_{1}, \mathscr{L}_{2}\right) \varrho\left(\mathscr{C}_{2}, \mathscr{C}_{3}\right)}{\varrho\left(\mathscr{L}_{3}, \mathscr{L}_{1}\right)} \sum_{\substack{\alpha \in \mathscr{L}_{2} /\left(\mathscr{L}_{2} \cap \mathscr{L}_{3}\right) \\ \beta \in \mathscr{L}_{3} /\left(\mathscr{L}_{3} \cap \mathscr{L}_{1}\right) \\ \alpha+\beta \in \mathscr{L}_{1}}} \chi_{p}(1 / 2 . \mathscr{S}(\alpha, \beta))
$$


Proof leans upon the formula (2) for a canonical intertwining operator. Let $f \in H\left(\mathscr{L}_{1}\right)$, then we have

$$
\begin{aligned}
\mathscr{F} f(u)= & \varrho\left(\mathscr{L}_{1}, \mathscr{L}_{2}\right) \varrho\left(\mathscr{L}_{2}, \mathscr{L}_{3}\right) \varrho\left(\mathscr{L}_{3}, \mathscr{L}_{1}\right) \sum_{\substack{\gamma \in \mathscr{L}_{1} /\left(\mathscr{L}_{3} \cap \mathscr{L}_{1}\right) \\
\beta \in \mathscr{L}_{3} /\left(\mathscr{L}_{2} \cap \mathscr{L}_{3}\right) \\
\alpha \in \mathscr{L}_{2} /\left(\mathscr{L}_{1} \cap \mathscr{L}_{2}\right)}} \chi_{p}(1 / 2 \mathscr{B}(\gamma, u) \\
& +1 / 2 \mathscr{B}(\beta, u+\gamma)+1 / 2 \mathscr{B}(\alpha, u+\beta+\gamma)) f(u+\alpha+\beta+\gamma) \\
= & \varrho\left(\mathscr{L}_{1}, \mathscr{L}_{2}\right) \varrho\left(\mathscr{L}_{2}, \mathscr{L}_{3}\right) \varrho\left(\mathscr{L}_{3}, \mathscr{L}_{1}\right) \sum_{\substack{\gamma \in \mathscr{L}_{1} /\left(\mathscr{L}_{3} \cap \mathscr{L}_{1}\right) \\
\beta \in \mathscr{L}_{3} /\left(\mathscr{L}_{2} \cap \mathscr{L}_{3}\right) \\
\alpha \in \mathscr{L}_{2} /\left(\mathscr{L}_{1} \cap \mathscr{L}_{2}\right)}} \\
& \times \chi_{p}(1 / 2 \mathscr{B}(\alpha, \beta)+\mathscr{B}(\alpha+\beta, \gamma)) f(u+\alpha+\beta) .
\end{aligned}
$$

By using the last formula for $f=\phi_{\mathscr{L}_{1}}$ we get the needed formula:

$$
\begin{aligned}
\mu\left(\mathscr{L}_{1}, \mathscr{L}_{2}, \mathscr{L}_{3}\right) & =\left(\mathscr{F} \phi_{\mathscr{L}_{1}}, \phi_{\mathscr{L}_{1}}\right)_{H\left(\mathscr{L}_{1}\right)} \\
= & \frac{\varrho\left(\mathscr{L}_{1}, \mathscr{L}_{2}\right) \varrho\left(\mathscr{L}_{2}, \mathscr{L}_{3}\right)}{\varrho\left(\mathscr{L}_{3}, \mathscr{L}_{1}\right)} \sum_{\substack{\alpha \in \mathscr{L}_{2} /\left(\mathscr{L}_{2} \cap \mathscr{L}_{3}\right) \\
\beta \in \mathscr{L}_{3} /\left(\mathscr{L}_{3} \cap \mathscr{S}_{1}\right) \\
\alpha+\beta \in \mathscr{L}_{1}}} \chi_{p}(1 / 2 \mathscr{B}(\alpha, \beta))
\end{aligned}
$$

Proposition 4 shows that the Maslov index of a triple of selfdual lattices does depend on only the "relative positions" of lattices, although in its definition one uses a representation of the Heisenberg group.

Proposition 5. Let $\mathscr{L}_{1}, \mathscr{L}_{2}, \mathscr{L}_{3}, \mathscr{L}_{4} \in \Lambda$. The following statements are valid.

(i) $\mu\left(\mathscr{L}_{1}, \mathscr{L}_{2}, \mathscr{L}_{3}\right)=\mu\left(g \mathscr{L}_{1}, g \mathscr{L}_{2}, g \mathscr{L}_{3}\right)$ for all $g \in S p(\mathscr{V})$;

(ii) $\mu\left(\mathscr{L}_{1}, \mathscr{L}_{2}, \mathscr{L}_{3}\right)=1$ if at least two lattices in the triple coincide;

(iii) $\mu\left(\mathscr{L}_{1}, \mathscr{L}_{2}, \mathscr{L}_{3}\right)$ remains the same under an even permutation of lattices in the triple and transfers to a conjugate expression under an odd one;

(iv) the following cocycle relation holds:

$$
\mu\left(\mathscr{L}_{1}, \mathscr{L}_{2}, \mathscr{L}_{3}\right) \mu\left(\mathscr{L}_{1}, \mathscr{L}_{3}, \mathscr{L}_{4}\right)=\mu\left(\mathscr{L}_{2}, \mathscr{L}_{3}, \mathscr{L}_{4}\right) \mu\left(\mathscr{L}_{2}, \mathscr{L}_{4}, \mathscr{L}_{1}\right)
$$

Proof. (i) follows directly from the explicit formula for $\mu$ (Proposition 4). The statement (ii)-(iv) one proves in a similar manner immediately from the definition of $\mu$. Let us prove the statement (iv). From the definition of the Maslow index we have:

$$
\begin{aligned}
\mu\left(\mathscr{L}_{1}, \mathscr{L}_{2}, \mathscr{L}_{3}\right) \mu\left(\mathscr{L}_{1}, \mathscr{L}_{3}, \mathscr{L}_{4}\right) \mathrm{Id} \\
=F_{\mathscr{L}_{1}, \mathscr{L}_{4}} F_{\mathscr{L}_{4}, \mathscr{L}_{3}} F_{\mathscr{L}_{3}, \mathscr{L}_{2}} F_{\mathscr{L}_{2}, \mathscr{L}_{1}}=F_{\mathscr{L}_{2}, \mathscr{L}_{1}}^{-1}\left(F_{\mathscr{L}_{2}, \mathscr{H}_{1} 1} F_{\mathscr{L}_{1}, \mathscr{L}_{4}} F_{\mathscr{L}_{4}, \mathscr{S}_{2}}\right) \\
\quad \times\left(F_{\mathscr{L}_{2}, \mathscr{L}_{4}} F_{\mathscr{L}_{4}, \mathscr{L}_{3}} F_{\mathscr{L}_{3}, \mathscr{L}_{2}}\right) F_{\mathscr{L}_{2}, \mathscr{L}_{1}}=\mu\left(\mathscr{L}_{2}, \mathscr{L}_{3}, \mathscr{L}_{4}\right) \mu\left(\mathscr{L}_{2}, \mathscr{L}_{4}, \mathscr{L}_{1}\right) \mathrm{Id} .
\end{aligned}
$$

\section{Calculations of the Maslov Index}

Let us remind that any $x \in \mathbb{Q}_{p}^{*}$ can be uniquely represented in the following form:

$$
x=p^{\operatorname{ord}_{p}(x)} \varepsilon(x)
$$


where $\operatorname{ord}_{p}: \mathbb{Q}_{p}^{*} \rightarrow \mathbb{Z}$ and $|x|_{p}=p^{-\operatorname{ord}_{p}(x)} ; \varepsilon: \mathbb{Q}_{p}^{*} \rightarrow \mathbb{Z}_{p}^{*}$ and $\varepsilon(x)=x_{0}+x_{1} p+\ldots$, $x_{j}=0,1, \ldots, p-1, x_{0} \neq 0$. Fractional part $\{x\}_{p}$ equals 0 if $x \in \mathbb{Z}_{p}$ and for $x \notin \mathbb{Z}_{p}$ is defined by the formula

$$
\{x\}_{p}=p^{\operatorname{ord}_{p}(x)}\left(x_{0}+x_{1} p+\ldots+x_{-\operatorname{ord}_{p}(x)-1} p^{-\operatorname{ord}_{p}(x)-1}\right) .
$$

Let $\lambda_{p}: \mathbb{Q}_{p} \rightarrow \mathbb{T}$ be a function defined by the formula (see [VV]):

$$
\begin{aligned}
& \lambda_{p}(0)=1 \\
& \lambda_{p}(x)= \begin{cases}1, & \operatorname{ord}_{p}(x)=2 k, k \in \mathbb{Z}, \\
\left(\frac{\varepsilon(x)}{p}\right), & \operatorname{ord}_{p}(x)=2 k+1, k \in \mathbb{Z}, p \equiv 1(\bmod 4), \\
i\left(\frac{\varepsilon(x)}{p}\right), & \operatorname{ord}_{p}(x)=2 k+1, k \in \mathbb{Z}, p \equiv 3(\bmod 4),\end{cases}
\end{aligned}
$$

where $\left(\frac{\varepsilon(x)}{p}\right)$ is the Legendre symbol of a $p$-adic unit $\varepsilon(x) \in \mathbb{Z}_{p}^{*}$. This function has the following properties.

Lemma 1. Function $\lambda_{p}$ has the properties:

(i) $\lambda_{p}(-x)=\overline{\lambda_{p}(x)}$;

(ii) $\lambda_{p}\left(a^{2} x\right)=\lambda_{p}(x), a \in \mathbb{Q}_{p}^{*}$;

(iii) $\lambda_{p}(x) \lambda_{p}(y)=\lambda_{p}\left(\frac{x+y}{x y}\right) \lambda_{p}(x+y)$;

(iv) $\lambda_{p}(x) \lambda_{p}(y)=(x, y) \lambda_{p}(x y)$, where $(x, y)$ is the Hilbert symbol.

Proof. For the proof of the properties (i)-(iii) see [VV]. Taking into account that $\lambda_{p}(x)=1$ for $x \in \mathbb{Z}_{p}^{*}$, statement (ii) and the symmetry of (iv) it is sufficient to check (iv) for the cases $x=y=p, x=y=\eta p, x=p, y=\eta p$, where $\eta \in \mathbb{Z}_{p}^{*},\left(\frac{\eta}{p}\right)=-1$ that can be done by direct calculations.

From the definition of $\lambda_{p}$ it is easy to make out the connection of this function with the Gauss sum

$$
\sum_{k=0}^{p^{n}-1} \exp \left(2 \pi i a \frac{k^{2}}{p^{n}}\right)=p^{n / 2} \lambda_{p}\left(a p^{n}\right)
$$

where $a \in \mathbb{Z}, n \in \mathbb{Z}_{\geq 0}$ and $a$ is not divisible by $p$.

Let $m, n \in \mathbb{Z}, \mu, \nu \in \mathbb{Q}_{p}$ and $\{e, f\}$ be a symplectic basis of $(\mathscr{V}, \mathscr{B})$. We consider now the following triple of selfdual lattices in $(\mathscr{T}, \mathscr{B})$ :

$$
\begin{aligned}
& \mathscr{L}_{1}=\mathbb{Z}_{p} e \oplus \mathbb{Z}_{p} f, \\
& \mathscr{L}_{2}=\mathbb{Z}_{p} p^{m} e \oplus \mathbb{Z}_{p}\left(\mu p^{m} e+p^{-m} f\right), \\
& \mathscr{L}_{3}=\mathbb{Z}_{p} p^{n} e \oplus \mathbb{Z}_{p}\left(\nu p^{n} e+p^{-n} f\right) .
\end{aligned}
$$

As it is evident from the foregoing the Maslov index of these triples can be represented as function of $m, n, \mu$ and $\nu$, that is $\mu\left(\mathscr{L}_{1}, \mathscr{L}_{2}, \mathscr{L}_{3}\right)=M(m, \mu ; n, \nu)$ for some function $M:\left(\mathbb{Z} \times \mathbb{Q}_{p}\right) \times\left(\mathbb{Z} \times \mathbb{Q}_{p}\right) \rightarrow \mathbb{T}$. The explicit formulas for the function $M$ in simplest cases is given by the following theorem. 
Theorem. The following formulas are valid:

$$
\begin{aligned}
& M(m, 0 ; n, 0)=1 \text { for all } m, n \in \mathbb{Z} ; \\
& M(m, 0 ; 0, \nu)= \begin{cases}1, & m \geq 0 \text { or } \nu \in \mathbb{Z}_{p}, \\
\lambda_{p}(-\nu), & m<0,1<|\nu|_{p}<p^{-2 m}, \\
1, & m<0, p^{-2 m} \leq|\nu|_{p}\end{cases} \\
& M(0, \mu ; 0, \nu)= \begin{cases}1, \quad \mu \in \mathbb{Z}_{p} \text { or } \nu \in \mathbb{Z}_{p} \text { or } \quad \mu-\nu \in \mathbb{Z}_{p}, \\
\lambda_{p}(\mu \nu(\mu-\nu)) \text { in other cases. }\end{cases}
\end{aligned}
$$

Proof. Since $\left|\mu\left(\mathscr{L}_{1}, \mathscr{L}_{2}, \mathscr{L}_{3}\right)\right|=1$, then all calculations can be carried out up to some real positive factor and instead of the equality sign we shall write the sign $\sim$. By virtue of Proposition 4 and the last remark we have

$$
M(m, \mu, n, \nu) \sim \sum_{\substack{\alpha \in \mathscr{L}_{1} /\left(\mathscr{L}_{2} \cap \mathscr{L}_{3}\right) \\ \beta \in \mathscr{L}_{3} /\left(\mathscr{L}_{3} \cap \mathscr{L}_{1}\right) \\ \alpha+\beta \in \mathscr{L}_{1}}} \chi_{p}(1 / 2 \mathscr{\beta}(\alpha, \beta)) .
$$

(i) Taking into account Proposition 5 (ii) it is sufficient to consider the case $m \neq 0$, $m \neq n, n \neq 0$. Besides that we can reduce the general case to the case of $m>n$, $m>0$ by means of changes of order of lattices in the triple and transformation of basis $e \rightarrow f, f \rightarrow-e$ if it is necessary. Since $\alpha \in \mathscr{L}_{2}$ and $\beta \in \mathscr{L}_{3}$ they can be represented in the following form:

$$
\begin{gathered}
\alpha=\alpha_{1} p^{m} e+\alpha_{2} p^{-m} f, \\
\beta=\beta_{1} p^{n} e+\beta_{2} p^{-n} f,
\end{gathered}
$$

where $\alpha_{1}, a_{2}, \beta_{1}, \beta_{2} \in \mathbb{Z}_{p}$. As $p^{m} \alpha_{1} \in \mathbb{Z}_{p}$ if $m>0$ and $\alpha_{1} \in \mathbb{Z}_{p}$ then the condition $\alpha+\beta \in \mathscr{L}_{1}$ has the form:

$$
p^{n} \beta_{1} \in \mathbb{Z}_{p}, \quad p^{-m} \alpha_{2}+p^{-n} \beta_{2} \in \mathbb{Z}_{p} .
$$

Since $\chi_{p}$ is of rank 0 and taking into account the condition $m-n>0$ and the formula (8) we get:

$$
\begin{aligned}
\chi_{p}(\mathscr{B}(\alpha, \beta)) & =\chi_{p}\left(p^{m-n} \alpha_{1} \beta_{2}-p^{n-m} \alpha_{2} \beta_{1}\right)=\chi_{p}\left(-p^{n-m} \alpha_{2} \beta_{1}\right) \\
& =\chi_{p}\left(-p^{n} \beta_{1}\left(p^{-n} \beta_{2}+p^{-m} \alpha_{2}-p^{-n} \beta_{2}\right)\right) \\
& =\chi_{p}\left(-p^{n} \beta_{1}\left(p^{-n} \beta_{2}+p^{-m} \alpha_{2}\right)+\beta_{1} \beta_{2}\right)=1
\end{aligned}
$$

and therefore $M(m, 0 ; n, 0)=1$ for all $m, n \in \mathbb{Z}$.

(ii) Taking into account Proposition 1 and 5 (ii) it is sufficient to consider the case $m \neq 0, \nu \notin \mathbb{Z}_{p}$. Let $\alpha \in \mathscr{L}_{2}$ and $\beta \in \mathscr{L}_{3}$. Then we have

$$
\begin{gathered}
\alpha=\alpha_{1} p^{m} e+\alpha_{2} p^{-m} f, \\
\beta=\beta_{1} e+\beta_{2}(\nu e+f),
\end{gathered}
$$

where $\alpha_{1}, \alpha_{2}, \beta_{1}, \beta_{2} \in \mathbb{Z}_{p}$. The condition $\alpha+\beta \in \mathscr{L}_{1}$ has the form:

$$
p^{m} \alpha_{1}+\nu \beta_{2} \in \mathbb{Z}_{p}, \quad p^{-m} \alpha_{2} \in \mathbb{Z}_{p} .
$$


Since of $\chi_{p}$ is a character of rank 0 and taking into account the formula (9) we get:

$$
\begin{aligned}
\chi_{p}(\mathscr{B}(\alpha, \beta)) & =\chi_{p}\left(p^{m} \alpha_{1} \beta_{2}-p^{-m} \nu \alpha_{2} \beta_{2}\right) \\
& =\chi_{p}\left(p^{m} \alpha_{1} \beta_{2}-p^{-m} \alpha_{2}\left(p^{m} \alpha_{1}+\nu \beta_{2}-p^{m} \alpha_{1}\right)\right) \\
& =\chi_{p}\left(p^{m} \alpha_{1} \beta_{2}-p^{-m} \alpha_{2}\left(p^{m} \alpha_{1}+\nu \beta_{2}\right)+\alpha_{1} \alpha_{2}\right)=\chi_{p}\left(p^{m} \alpha_{1} \beta_{2}\right) .
\end{aligned}
$$

If $m \geq 0$ then as it follows from $(10) \chi_{p}(\mathscr{B}(\alpha, \beta))=1$ and $M(m, 0 ; 0, \nu)=1$. Let now $m<0$ and $|\nu|_{p} \geq p^{-2 m}$, that is $\operatorname{ord}_{p}(\nu) \leq 2 m$. By virtue of (9) and (10) we have

$$
\begin{aligned}
\chi_{p}(\mathscr{B}(\alpha, \beta)) & =\chi_{p}\left(p^{m-\operatorname{ord}_{p}(\nu)} \varepsilon^{-1}(\nu) \alpha_{1}\left(p^{m} \alpha_{1}+\nu \beta_{2}-p^{m} \alpha_{1}\right)\right) \\
& =\chi_{p}\left(p^{m-\operatorname{ord}_{p}(\nu)} \varepsilon^{-1}(\nu) \alpha_{1}\left(p^{m} \alpha_{1}+\nu \varphi_{2}\right)-p^{2 m-\operatorname{ord}_{p}(\nu)} \varepsilon^{-1}(\nu) \alpha_{1}^{2}\right) \\
& =\chi_{p}\left(-p^{2 m-\operatorname{ord}_{p}(\nu)} \varepsilon^{-1}(\nu) \alpha_{1}^{2}\right)=1
\end{aligned}
$$

and $M(m, 0 ; 0, \nu)=1$. In the last case $m<0$ and $1<|\nu|_{p}<p^{-2 m}$ the proof is given below for the case $1<|\nu|_{p} \leq p^{-m}$ (the case $p^{-m}<|\nu|_{p}<p^{-2 m}$ one considers analogously). Let $a$ and $b$ denote $\alpha_{1}$ and $\beta_{2}$ respectively, $n$ denotes $\operatorname{ord}_{p}(\nu)$ and $\varepsilon$ denotes $\varepsilon(\nu)$. As any $x \in \mathbb{Z}_{p}$ can be represented in the form

$$
x=x_{0}+x_{1} p+x_{2} p^{2}+\ldots, \quad x_{j}=0,1, \ldots, p-1,
$$

then the condition (9) takes the form

$$
p^{m}\left(a_{0}+a_{1} p+\ldots\right)+p^{n}\left(b_{0}+b_{1} p+\ldots\right) \varepsilon \in \mathbb{Z}_{p} .
$$

From the last formula we get that the formula (9) is equivalent to the set of equations:

$$
\begin{gathered}
a_{0}=a_{1}=\ldots=a_{n-m-1}=0, \\
a_{n-m}+(b \varepsilon)_{0}=0, \\
\vdots \\
a_{-m-1}+(b \varepsilon)_{-n-1}=0,
\end{gathered}
$$

thus from (10) we have

$$
\begin{aligned}
\chi_{p}(\mathscr{B}(\alpha, \beta))= & \chi_{p}\left(p^{n}\left(a_{n-m}+a_{n-m+1} p+\ldots\right)\left(b_{0}+b_{1} p+\ldots\right)\right) \\
= & \chi_{p}\left(-p^{n}\left((b \varepsilon)_{0}+(b \varepsilon)_{1} p+\ldots+(b \varepsilon)_{-n-1} p^{-n-1}\right)\right. \\
& \left.\times\left(b_{0}+b_{1} p+\ldots+b_{-n-1} p^{-n-1}\right)\right) \\
= & \chi_{p}\left(-p^{n}\left(b_{0}+b_{1} p+\ldots+b_{-n-1} p^{-n-1}\right)^{2} \eta\right)
\end{aligned}
$$

where $\eta=\varepsilon_{0}+\varepsilon_{1} p+\ldots+\varepsilon_{-n-1} p^{-n-1}$. It is easy to see that the set $\mathscr{L}_{3} \cap \mathscr{L}_{1}$ has the form:

$$
\mathscr{L}_{3} \cap \mathscr{L}_{1}=\left\{\beta_{1} e+\beta_{2}(\nu e+f), \beta_{1} \in \mathbb{Z}_{p}, \nu \beta_{2} \in \mathbb{Z}_{p}\right\},
$$

and from the last formula and (11) we have

$$
M(m, 0 ; 0, \nu) \sim \sum_{b_{0}, b_{1}, \ldots, b_{-n-1}=0}^{p-1} \chi_{p}\left(-p^{n} \eta\left(b_{0}+\ldots+b_{-n-1} p^{-n-1}\right)^{2}\right),
$$


whence it follows that

$$
M(m, 0 ; 0, \nu) \sim \sum_{k=0}^{p^{-n}-1} \exp \left(-2 \pi i \eta \frac{k^{2}}{p^{-n}}\right) .
$$

(Here we use the explicit form for the character $\chi_{p}(\xi)=\exp \left(2 \pi i\{\xi\}_{p}\right)$. Taking into account the formula (7) we get the needed formula $M(m, 0 ; 0, \nu)=\lambda_{p}\left(-p^{-n} \eta\right)=$ $\lambda_{p}(-\nu)$.

(iii) Taking into account Propositions 1 and 5 (ii) it is sufficient to consider the case $\mu \notin \mathbb{Z}_{p}, \mu-\nu \notin \mathbb{Z}_{p}, \nu \notin \mathbb{Z}_{p}$. We present here the proof only for the case of $|\mu|_{p} \neq|\nu|_{p}$, otherwise (iii) can be proved analogously. By the symmetry we can suppose that $|\nu|_{p}<|\mu|_{p}$. Let $\alpha \in \mathscr{L}_{2}, \beta \in \mathscr{L}_{3}$, then

$$
\begin{gathered}
\alpha=\alpha_{1} e+\alpha_{2}(\mu e+f), \\
\beta=\beta_{1} e+\beta_{2}(\nu e+f),
\end{gathered}
$$

where $\alpha_{1}, \alpha_{2}, \beta_{1}, \beta_{2} \in \mathbb{Z}_{p}$. The condition $\alpha+\beta \in \mathscr{L}_{1}$ takes the form:

$$
\mu \alpha_{2}+\nu \beta_{2} \in \mathbb{Z}_{p}
$$

Since the rank of $\chi_{p}$ equals 0 we have:

$$
\chi_{p}(\mathscr{B}(\alpha, \beta))=\chi_{p}\left(\mu \alpha_{2} \beta_{2}-\nu \alpha_{2} \beta_{2}\right)=\chi_{p}\left((\mu-\nu) \alpha_{2} \beta_{2}\right) .
$$

Let $\operatorname{ord}_{p}(\mu)-m$, ord $(\nu)=-n, \alpha_{2}=a, \beta_{2}=b$. As for the proof of the statement (ii) from the formula (12) we get:

$$
p^{-m} \varepsilon(\mu)\left(a_{0}+a_{1} p+\ldots\right)+p^{-n} \varepsilon(\nu)\left(b_{0}+b_{1} p+\ldots\right) \in \mathbb{Z}_{p} .
$$

In the case of $m>n \geq 1$ from the last formula we have:

$$
\begin{gathered}
\left(\varepsilon \left(\mu(a)_{0}=(\varepsilon(\mu) a)_{1}=(\varepsilon(\mu) a)_{m-n-1}=0,\right.\right. \\
(\varepsilon(\mu) a)_{m-n}+(\varepsilon(\nu) b)_{0}=0, \\
\vdots \\
(\varepsilon(\mu) a)_{m-1}+(\varepsilon(\nu) b)_{n-1}=0 .
\end{gathered}
$$

As for the proof of (ii) from (12) and (13) we have:

$$
\chi_{p}(\mathscr{B}(\alpha, \beta))=\chi_{p}\left(-(\mu-\nu) \frac{\varepsilon(\nu)}{\varepsilon(\mu)} p^{m-n}\left(b_{0}+b_{1} p+\ldots+b_{n-1} p^{n-1}\right)^{2}\right) .
$$

Since $\operatorname{ord}_{p}(\mu-\nu)=\operatorname{ord}_{p}(\mu)$ from the last formula we obtain:

$$
\chi_{p}(\mathscr{B}(\alpha, \beta))=\chi_{p}\left(p^{-n} \eta\left(b_{0}+b_{1} p+\ldots+b_{n-1} p^{n-1}\right)^{2}\right),
$$

where

$$
\eta=\left(\varepsilon(\nu-\mu) \frac{\varepsilon(\nu)}{\varepsilon(\mu)}\right)_{0}+\left(\varepsilon(\nu-\mu) \frac{\varepsilon(\nu)}{\varepsilon(\mu)}\right)_{1} p+\ldots+\left(\varepsilon(\nu-\mu) \frac{\varepsilon(\nu)}{\varepsilon(\mu)}\right)_{n} p^{n-1} .
$$

The set $\mathscr{L}_{3} \cap \mathscr{L}_{1}$ has the form

$$
\mathscr{L}_{3} \cap \mathscr{L}_{1}=\left\{\beta_{1} e+\beta_{2}(\nu e+f), \beta_{1} \in \mathbb{Z}_{p}, \nu \beta_{2} \in \mathbb{Z}_{p}\right\}
$$


and as for the proof of (ii) we have:

$$
M(0, \mu ; 0, \nu)=\lambda_{p}\left(p^{n} \eta\right) .
$$

Taking into account the properties of the function $\lambda_{p}$ and the relation $\operatorname{ord}_{p}(\nu-\mu)=$ $\operatorname{ord}_{p}(\mu)$ we derive from the last formula:

$$
\begin{aligned}
M(0, \mu ; 0, \nu) & =\lambda_{p}\left(p^{n} \varepsilon(\nu-\mu) \frac{\varepsilon(\nu)}{\varepsilon(\mu)}\right) \\
& =\lambda_{p}\left(p^{n} \varepsilon(\nu) p^{m} \varepsilon(\mu) p^{m} \varepsilon(\nu-\mu)\right)=\lambda_{p}(\nu(\nu-\mu)) .
\end{aligned}
$$

The proved theorem makes possible to calculate the Maslov index in the general case. By Proposition 1 for an arbitrary triple $\left(\mathscr{L}_{1}, \mathscr{L}_{2}, \mathscr{L}_{3}\right)$ of selfdual lattices there is a symplectic basis $\{e, f\}$ wherein

$$
\begin{aligned}
& \mathscr{C}_{1}=\mathbb{Z}_{p} e \oplus \mathbb{Z}_{p} f, \\
& \mathscr{L}_{2}=\mathbb{Z}_{p} p^{m} e \oplus \mathbb{Z}_{p} p^{-m} f, \\
& \mathscr{L}_{3}=\mathbb{Z}_{p} p^{n} e \oplus \mathbb{Z}_{p}\left(\nu p^{n} e+p^{-n} f\right),
\end{aligned}
$$

where $m \in \mathbb{Z}_{\geq 0}, n \in \mathbb{Z}, \nu \in \mathbb{Q}_{p}$. Therefore the Maslov index of this triple is given by the relation

$$
\mu\left(\mathscr{L}_{1}, \mathscr{L}_{2}, \mathscr{L}_{3}\right)=M(m, 0 ; n, \nu) .
$$

Let $\mathscr{L}_{4}=\mathbb{Z}_{p} p^{n} e \oplus \mathbb{Z}_{p} p^{-n} f$. In the symplectic basis $\left\{\tilde{e}=p^{n} e, \tilde{f}=p^{-n} f\right\}$ we have

$$
\begin{aligned}
& \mathscr{L}_{1}=\mathbb{Z}_{p} p^{-n} \tilde{e} \oplus \mathbb{Z}_{p} p^{n} \tilde{f}, \\
& \mathscr{L}_{2}=\mathbb{Z}_{p} p^{m-n} \tilde{e} \oplus \mathbb{Z}_{p} p^{n-m} \tilde{f}, \\
& \mathscr{L}_{3}=\mathbb{Z}_{p} \tilde{e} \oplus \mathbb{Z}_{p}(\nu \tilde{e}+\tilde{f}), \\
& \mathscr{L}_{4}=\mathbb{Z}_{p} \tilde{e} \oplus \mathbb{Z}_{p} \tilde{f} .
\end{aligned}
$$

Taking into account Proposition 5(i), (iii), (iv) we have

$$
\begin{aligned}
\mu\left(\mathscr{S}_{1}, \mathscr{L}_{2}, \mathscr{L}_{3}\right) & =\bar{\mu}\left(\mathscr{C}_{1}, \mathscr{L}_{3}, \mathscr{L}_{4}\right) \mu\left(\mathscr{L}_{2}, \mathscr{L}_{3}, \mathscr{L}_{4}\right) \mu\left(\mathscr{L}_{2}, \mathscr{L}_{4}, \mathscr{L}_{1}\right) \\
& =\bar{M}(-m, 0 ; 0, \nu) M(m-n, 0 ; 0, \nu) M(-n, 0 ; m-n, 0) .
\end{aligned}
$$

By virtue of the theorem and the last formula the following corollary is valid.

Corollary. For the lattices $\mathscr{L}_{1}, \mathscr{L}_{2}, \mathscr{L}_{3}$ of the form (14) we have

$$
\mu\left(\mathscr{S}_{1}, \mathscr{S}_{2}, \mathscr{S}_{3}\right)= \begin{cases}1, & m=0 \text { or } \nu \in \mathbb{Z}_{p} \quad \text { of } n \leq 0, \\ \lambda_{p}(\nu), & 0<n \leq m, 1<|\nu|_{p}<p^{2 n}, \\ 1, & 0<n \leq m, p^{2 n} \leq|\nu|_{p}, \\ 1, & m<n, 1<|\nu|_{p}<p^{2(n-m)}, \\ \lambda_{p}(\nu), & m<n, p^{2(n-m)} \leq|\nu|_{p}<p^{2 n}, \\ 1, & m<n, p^{2 n} \leq|\nu|_{p} .\end{cases}
$$

Acknowledgements. I would like to thank I. Volovich for helpful discussions and the referee for valuable comments. 


\section{References}

[C] Cartier, P.: Quantum mechanical commutation relations and theta-functions. Proc. Sympos. Pure Math. 9, 361-383 (1966)

[LV] Lion, G., Vergne, M.: The Weil representation, Maslov index and theta-series. Boston: Birkhäuser 1980

[Ma] Maslov, V.P.: Aymptotic methods and perturbation theory. Moscow: Nauka 1988

[Me] Meurice, Y.: Quantum mechanics with $p$-adic numbers. Int. J. Math. Phys. A 4, 5133-5147 (1989)

[MH] Milnor, J., Husemoller, D.: Symmetric bilinear forms. Berlin, Heidelberg, New York: Springer 1973

[PR] Platonov, V.P., Rapinchuk, A.S.: Algebraic groups and number theory. Moscow: Nauka 1990

[R] Ruelle, P., Thiran, E., Verstegen, D., Weyers, J.:, Quantum mechanics on $p$-adic fields. J. Math. Phys. 30, 2854-2861 (1989)

[VV] Vladimirov, V.S., Volovich, I.V.: p-Adic quantum mechanics. Commun. Math. Phys. 123, 659-676 (1989)

[V] Volovich, I.V.: p-Adic strings. Class. Quant. Grav. 4, L 83-L 87 (1987)

[W1] Weil, A.: Basic number theory. Berlin, Heidelberg, New York: Springer 1967

[W2] Weil, A.: Sur certains groupes d'operateurs unitaires. Acta Mathem. 111, 143-211 (1964)

Communicated by H. Araki 